\title{
Comparative Study of the Psychological Well-Being of Healthy Volunteers, Paralympic Athletes and Non-Athletes with Spinal Cord Injury, Assessed by Short Form Survey (SF-36)
}

\author{
Mirna Clemente $^{1^{*}}$, Marilis Dallarmi Miguel ${ }^{1}$, Karina Bettega Felipe ${ }^{2}$, Maria Gisele Santos ${ }^{3}$, \\ Ruth Eugenia Cidade ${ }^{3}$, Darlan França Ciesielski Junior ${ }^{5}$, Luiz Claudio Fernandes ${ }^{4}$, Obdulio \\ Gomes Miguel, ${ }^{1}$, \\ ${ }^{1}$ Pharmaceutical Science Department, UFPR Brazil \\ ${ }^{2}$ Biochemical Department, UFPR \\ ${ }^{3}$ Physical Education Department UFPR \\ ${ }^{4}$ Physiology Department, UFPR. Brazil \\ ${ }^{5}$ Physical Educator, ADFP ( Physical Disability Association Of Parana)
}

*Corresponding Author: Mirna Clemente, Pharmaceutical Science Department, UFPR Brazil

\begin{abstract}
People with Spinal Cord Injury (SCI) are susceptible to several secondary problems, such as pressure ulcers, urinary infections, and a high risk of depression. Otherwise, it has been widely accepted that participation in sport may provide opportunities for people with disabilities, with the potential to promote quality of life demonstrated by psychological functioning. This study aimed to evaluate and compare the psychological well-being of Paralympic Athletes with SCI (PA), Non-Athletes with SCI (NA) and Healthy Volunteers $(H V)$, assessed by SF-36. This is a comparative, descriptive, and qualitative study. The protocol for carrying out this research followed the Standards for Reporting Qualitative Research (SRQR). After inclusion and exclusion criteria, forty people were asked to complete the informed consent form and the questionnaire. Then, they were divided into three groups: PA, NA, and HV. The data of this study demonstrated that the Vitality, Social Functioning, Role Emotional Limitation, Mental Health, and Mental Composite Score were higher in PA group, as compared to NA, and lower in Physical Functioning compared to HV group. Finally, strengthening the engagement in Paralympic sport among people with SCI is a promising strategy to promote psychological well-being and decrease susceptibility to several secondary problems in this vulnerable group.
\end{abstract}

Keywords: SF-36; Psychological Well-Being; Rehabilitation, Quality of Life, Paralympic Sport and Spinal Cord Injury

Abbreviations: BP: Bodily Pain; GH: General Health Status; HV: Healthy Volunteer; MCS: Mental composite score; MH: Mental Health; NA: Non-Athlete with Spinal Cord Injury; PA: Paralympic Athlete with Spinal Cord Injury; PCS: Physical Composite Score; PF: Physical Functioning; REL: Role Emotional Limitation; RPL: Role Physical Limitation; SF 36: Short Form Survey; SCI: Spinal Cord Injury; SF: Social Functioning; V: Vitality

\section{INTRODUCTION}

The spinal cord injury (SCI), the subject of this study, refers to any type of lesion (congenital or acquired) that occurs in the neural elements of the spinal cord. The classification depends on the level of the impairment. The term tetraplegia refers to the most severe form, and all limbs are affected, in the movement and paraplegia indicates a condition in which they are primarily affected the lower limbs [1].

People with spinal cord injury are susceptible to several secondary problems, such as pressure ulcers, urinary tract infections, spasticity, contractures, obesity and also difficulty in acceptance (leading to depression), low self-esteem and sexual dysfunction [2] [3] [4] [5]. Depression is one of the psychological conditions associated with SCI, which has been well studied [6]; this disease may increase comorbidities and show a negative impact on the quality of life [7]. One of the goals of the rehabilitation program is to improve the level of Health-Related to Quality of Life (HRQOL). 
Otherwise, it has been widely accepted that participation in sports can improve physical and mental health, decreasing anxiety, depression, and fatigue [8]. Paralympic sport or being engaged in sport may provide opportunities for people with disabilities, with the potential to promote the social model of disabilit3y and improve Health Related to Quality of Life (HRQL) demonstrated by psychological functioning [9] [10].

The interest in the sports participation on the psychological well-being of people with disabilities has been increasing [12]; since there was evidence that the prevalence of depression ranged around 9.7\% at five years after acquiring SCI [11], and this population has demonstrated a higher risk of depression than individuals in the other health conditions group [7].

In light of the above, the present study aimed to evaluate and compare physiological well-being assessed by the Short Form Survey (SF-36) on Paralympic athletes with SCI, comparing to Non-athletes with SCI and Healthy Volunteers. Future researches with different rehabilitation strategies on people with SCI or other disabilities are recommended. In addition, we suggest that government policies should encourage engagement in Paralympic sport among these people, being a promising strategy to promote mental health and reducing the prevalence of depression, as well improving their quality of life.

\section{MAterial \& Methods}

\subsection{Study Design}

This is a comparative, descriptive, and qualitative study. The local ethics committee (Federal University of Paraná- Brazil) under the number 2.294.303. The protocol for carrying out the study was developed, by following the Standards for Reporting Qualitative Research (SRQR) with 21 items [13].

\subsection{Participants}

People with disability and healthy volunteers from the Physical Disability Association of Parana (ADFP) from Curitiba, Parana, Brazil, were invited to participate in this study.

Inclusion criteria:

- 18 years old or older

- To be associated with the ADFP, as a member or volunteer for more than two years

- People with spinal cord injury from Paralympic sport and non-active

- Healthy and active volunteers

Exclusion criteria:

- Using depression or anxiolytic medicines

- Tetraplegia

\subsection{Procedures}

After inclusion and exclusion criteria, forty people were asked to complete the informed consent form and the questionnaire. Then, these individuals were divided into three groups: Paralympic athletes with spinal cord injury group, Non-athletes with spinal cord injury group and healthy active people group.

\subsection{Questionnaire}

This study used the Medical Outcomes Study 36-item Short Form Survey (SF-36) to measure the quality of life people with spinal cord injury and health people, validated in Brazil [14].

The Short Form Survey (SF-36) consists of 36 questions, comprising eight domains:

- Physical Functioning (PF); evaluates the limitations related to physical capacity;

- Role Physical Limitation (RPL); assesses the constraints of the type of work and connects the tasks of practical daily life;

- Bodily Pain (BP): evaluates the existence of pain, its intensity, and how much it limits day-to-day activities;

- General health status (GH); assesses the general health of the patient;

- Vitality (V); is related to vigor, energy, and fatigue;

International Journal of Sports and Physical Education (IJSPE)

Page $\mid 24$ 
- Social Functioning (SF); analyzes socialization;

- Role Emotional Limitation (REL): evaluates aspects of the psychological well-being of the patient;

- Mental health (MH): assesses the patient's anxiety, depression, behavior, and psychological wellbeing.

The first four domains can create the Physical Composite Score (PCS) and the last four the Mental Composite Score (MCS), which provides a measure of the overall effect of physical and psychological impairment. Scores for the SF-36 scales range between 0 and 100, with higher scores indicating better quality of life. This questionnaire has been demonstrated as one of the most trusted measures of quality of life on people with spinal cord injury [15] [16] [17].

\subsection{Statistics}

Analyses of Short Form SF-36 in each domain and summary were made, to investigate the quality of life, particularly the psychological well-being. Statistical analysis was carried out using the one-way analysis of variance (ANOVA) with Tukey's multiple comparison tests and/or Kruskal-Wallis test, followed by Dunn's test if the requirements for performing a parametric test were not satisfied. Results are demonstrated, as mean \pm S.E.M. Using GraphPad Prism Software (GraphPad Software, Inc. La Jolla, CA, USA, version 7.0), significance was considered at a $p<0.05$.

\section{RESUlTS \& DISCUSSION}

Initially, forty-five male people from the ADFP were invited to participate in this study, and five of them were not selected since they did not follow the inclusion and exclusion criteria, showed in figure 1. Finally, forty people were asked to complete; the informed consent form and the questionnaire, and then they were allocated in three groups:

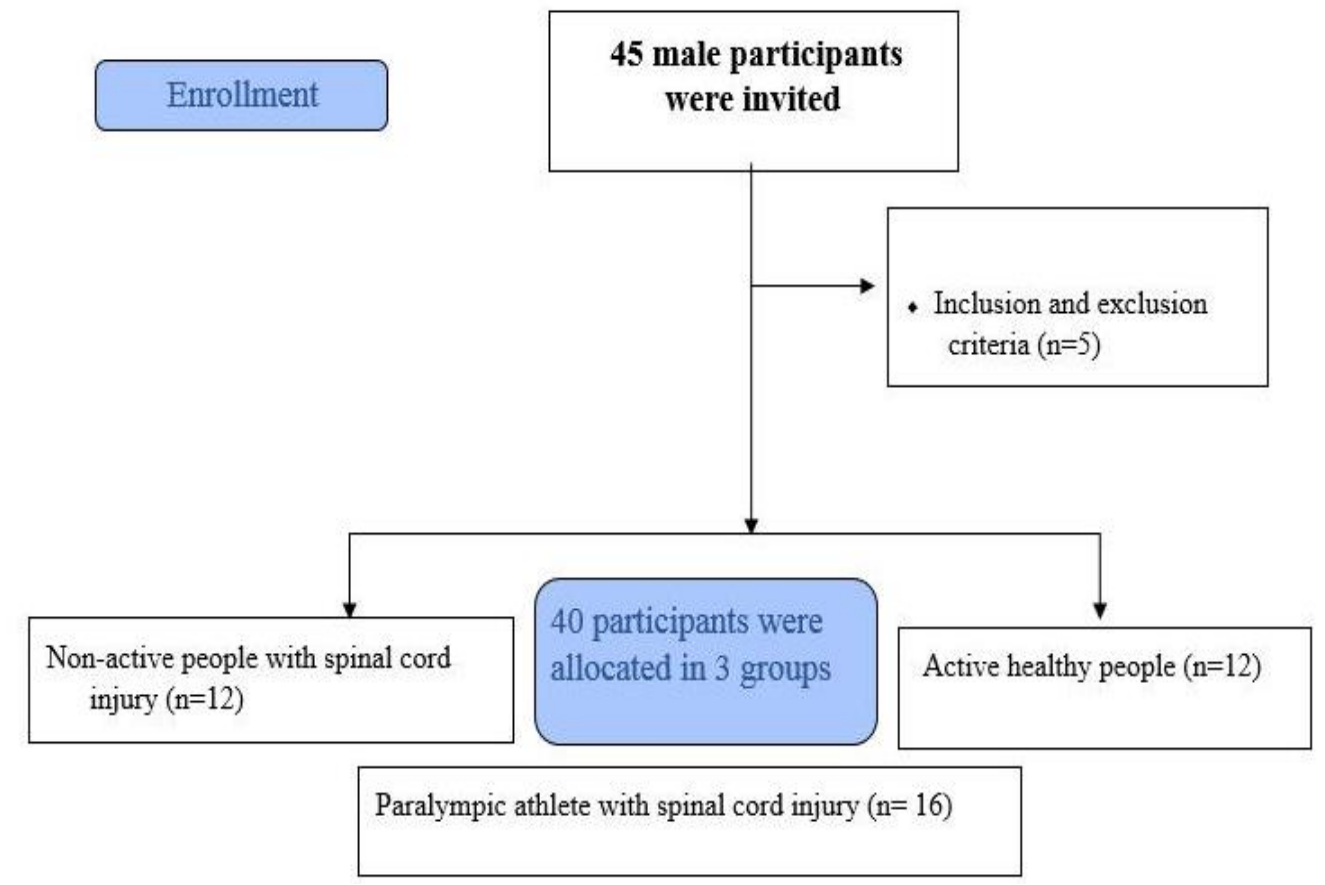

Figure1. Flow diagram of the enrollment of the study

Group 1: Paralympic athlete with spinal cord injury the average age was 37.56 .

Group 2: Non-athlete with spinal cord injury the average age was 35.83 .

Group 3: Active and healthy volunteers, the average age was 33.17.

The characteristics of three groups assessed by SF-36 questionnaire and the results, by PF, RP, BP, GH, VT, SF, RE, $138 \mathrm{MH}, \mathrm{PCS}, \mathrm{MCS}$, standard error of the mean (S.E.M). No statistically significant differences on RP, BP, GH, and PCS were demonstrated among the three groups. On PF, there were significant differences between the NA group and HV group (16.67 vs. 96.67), PA and HV (29.69 vs. 
96.67), and also PA and NA (29.69 vs. 16.67). On the domain of Vitality, statistical differences between NA and PA groups (58.75 vs. 81.06) were found. The SF domain the differences observed were between NA and PA (48.43 vs. 87.5), and NA and HV (48.43 vs. 92.71). The RE was statistically different between NA and PA group, and NA and HV group (50.83 vs. 89.58), (50.83 vs. 100) respectively. The Mental Health score on Paralympic athlete and non-athlete group (83 vs. 57.67) and Healthy volunteers and non-athlete group (76.67 vs. 57.67), were with significant differences. Finally, the Mental Composite Score showed a lower score and statistical difference between NA and PA group (53.92 vs. 85.29), and NA and HV group (53.92 vs. 84.12). All domains and the results were shown in table 1.

Table 1: Findings of Short Form Survey (SF 36) domains on the three groups.

\begin{tabular}{|l|l|l|l|l|l|l|}
\hline \multirow{3}{*}{$\begin{array}{c}\text { SF-36 } \\
\text { SCALES }\end{array}$} & \multicolumn{2}{|c|}{ Paralympic athletes with SCI } & \multicolumn{2}{c|}{ Non-Athletes with SCI } & \multicolumn{2}{c|}{ Healthy Volunteers } \\
\cline { 2 - 7 } & \multicolumn{1}{|c|}{ Group } & \multicolumn{1}{|c|}{ N= 16 } & Group & N= 12 & Group & \multicolumn{1}{c|}{ N= 12 } \\
\cline { 2 - 7 } & \multicolumn{1}{|c|}{ S.E.M } & \multicolumn{1}{|c|}{ Mean } & S. E.M. & Mean & S.E.M \\
\hline PF & 29.69 & $2,560 *$ & 16.67 & 1,28 & 96.67 & $1.54 * * * * *$ \\
\hline RP & 92 & 4.45 & 82.51 & 8.53 & 93.75 & 4.48 \\
\hline BP & 64,81 & 6.25 & 56,42 & 6,03 & 75,83 & 3,88 \\
\hline GH & 70.31 & 5.20 & 63.42 & 5.67 & 76.5 & 4.25 \\
\hline VT & 81.06 & $1.96^{*}$ & 58.75 & 7.15 & 67.08 & 6.10 \\
\hline SF & 87.5 & $4.56 *$ & 48.43 & 8.43 & 92.71 & $4.47 * *$ \\
\hline RE & 89.58 & $6.61 *$ & 50.83 & 14.85 & 100 & $0 * *$ \\
\hline MH & 83 & $2.08 *$ & 57.67 & 8.44 & 76.67 & $4.48 * *$ \\
\hline PCS & 64.2 & 12.91 & 54.76 & 13.84 & 85.69 & 5.52 \\
\hline MCS & 85.29 & $1.96 *$ & 53.92 & 2.53 & 84.12 & $7.48 * *$ \\
\hline
\end{tabular}

$* \mathrm{P}<0.05 \mathrm{PA}$ and $\mathrm{NA}, * * \mathrm{P}<0.05 \mathrm{NA}$ and $\mathrm{HV}, * * * \mathrm{P}<0.05 \mathrm{PA}$ and $\mathrm{HV}$.

The National SCI Statistical Center (NSCISC, 2015) estimates that about 12,000 people acquire spinal cord injuries each year in the United States. Globally, the incidence is the highest in males aged 18-32 years old [18]. Among the major causes are automobile accidents ( 39.2 percent), falls ( 28.3 percent), and acts of violence (14.6 percent), athletic injuries ( 8.2 percent), and other accidents ( 9.7 percent). In our study, the highest cause was a traffic accident (56 percent), and all the male participants with SCI were classified with paraplegia, which refers to impairment or loss of motor, sensory function in the thoracic, lumbar or sacral (but not cervical) [19].

Rehabilitation is the development of physical, psychological, and social parameters on people in general, in this case, mainly to paraplegic people. The rehabilitation process for trauma caused by SCI is long and expensive, starts with admission to hospital and stabilization of the patient's neurological state and is divided into acute, sub-acute, and chronic [19]. Therefore, Paralympic sport used as a rehabilitation program is increasingly serving to develop and maintain psychological functioning, promote a healthy, and increase life satisfaction of people with disabilities [10].

The present study aimed to evaluate and compare the psychological parameters, assessed by SF-36 short version, among the three chosen groups. We hypothesized that VT, SF, RE, MH and MCS, in people with SCI who were engaged to Paralympic sport would be higher than people with SCI not involved in any sport, and similar score to health people involved in sport, since the sport might show good benefits to people with SCI, in terms of the psychological well-being.

The first four domains, which represent the Physical domains and create the Physical Composite Score (PCS) and the RP, BP, GH, and PCS, did not demonstrate any statistical difference, and the characteristics of three groups assessed by SF-36 seem to be similar in these parameters. In contrast, the Physical Functioning was statistically different when healthy volunteers were compared to PA and NA group; it is not surprising that people with SCI have a much lower score in this area, corroborating to [20] [21] [22]. Moreover, the PA group had a higher rating as compared to the NA group. Since research indicates exercise may enhance functional independence and activities of daily living in people with SCI [23], the last results could be justified.

Otherwise, depression is not a natural process experienced after SCI, but this is a disorder which should be treated [19]. On the domains, which represent the psychological impairment, including depression symptoms, it was found statistical differences in the SF, RE, and MH when PA and HV 
group were compared to the NA group. The vitality score was also higher, but only between PA and NA group; when PA was compared to the HV group, the results did not show any significant difference.

Moreover, there is evidence that physical exercise and sports might bring benefits for people with disabilities on self-esteem, quality of life, and body image [24]. In addition, studies have shown the level of self-confidence with similar score among athletes with disabilities and athletes in the general population [21] [25]. In this study, MCS was statistically lower in non-athlete with SCI group, as compared to Paralympic and Healthy groups. Nonetheless, psychological variables related to engaging in competitive sport may have impacted the participants' motivation [26]. It might be the reason why the Paralympic group demonstrated similar scores to the active, healthy group since the Paralympic participants of this study are used to participate to National competition of Paralympic table tennis and basketball, and wheelchair fencing, 31.25\% of them to International competitions.

In light of the preceding discussion, the data support the importance of Paralympic sport in the life of people with spinal cord injury chosen in this study, since the psychological well-being was higher in the PA group as compared to NA group; demonstrated by other authors [27]. Therefore, this study was able to confirm that the Paralympic athletes and healthy volunteers group were more likely to have a similar experience on SF-36, in most of the domains, in this case, corroborating to [28].

This study demonstrated some limitations that might affect the results negatively. According to [29], depression after SCI has shown possible links to multiple indicators including aging, gender (female), and socioeconomic status, unfortunately, the only link demonstrated in this study was the sport against psychological disorder.

\section{CONCLUSION}

In conclusion, strengthening the engagement in Paralympic sport among people with spinal cord injury is a promising strategy to promote psychological well-being and decrease susceptible to several secondary problems in this vulnerable group. Further studies are recommended to consider the risk of bias demonstrated in this research, in addition, Paralympic sport as a rehabilitation program should be encouraged by the government policies, involving healthcare providers and people with SCI.

\section{ACKNOWLEDGMENTS}

This study was funded and supported by Federal University of Parana; Pharmaceutical Science Department, Physical Disability Association of Parana (ADFP). and also financed in part by the Coordination for the Improvement of Higher Education Personnel (CAPES) in Brazil- Financial code 001.

\section{REFERENCES}

[1] Winnick JP, Porretta DL. Adapted physical education and sport [Internet]. 2017. p. 328. Available from: https://www.worldcat.org/title/adapted-physical-education-and-sport/oclc/990549467

[2] Kinne S, Patrick DL, Doyle DL. Prevalence of Secondary Conditions among People with Disabilities. American Journal of Public Health. 2004; 94:443-5.

[3] Wilber N, Mitra M, Klein Walker D, Allen D, Meyers AR, Tupper P. Disability as a Public Health Issue: Findings and Reflections from the Massachusetts Survey of Secondary Conditions. Milbank Quarterly. 2002; 80:393-421.

[4] Ramos AS, Samsó J V. Specific aspects of erectile dysfunction in spinal cord injury. International Journal of Impotence Research. 2004;16:42-5.

[5] Huang T-S, Su T-C, Wang Y-H, Liang H-W, Wang T-D, Chen S-Y. Fasting Serum Levels of Adiponectin, Ghrelin, and Leptin in Men With Spinal Cord Injury. Archives of Physical Medicine and Rehabilitation. 2005; 86:1964-8.

[6] Fann JR, Bombardier CH, Richards JS, Tate DG, Wilson CS, Temkin N. Depression after spinal cord injury: Comorbidities, mental health service use, and adequacy of treatment. Archives of Physical Medicine and Rehabilitation [Internet]. Elsevier Inc.; 2011; 92:352-60. Available from: http://dx.doi.org/ 10.1016/j.apmr.2010.05.016

[7] Lim S, Shiue Y, Ho C, Yu S, Kao P, Wang J, et al. Anxiety and Depression in Patients with Traumatic Spinal Cord Injury: A Nationwide Population-Based Cohort Study. PLoS ONE. 2017;1-14.

[8] Shapiro DR, Malone LA. Quality of life and psychological effect related to sport participation in children and youth athletes with physical disabilities: A parent and athlete perspective. Disability and Health Journal [Internet]. Elsevier Inc; 2016; 9:385-91. Available from: http://dx.doi.org/10.1016/j.dhjo.2015. 11.007 
Comparative Study of the Psychological Well-Being of Healthy Volunteers, Paralympic Athletes and NonAthletes with Spinal Cord Injury, Assessed by Short Form Survey (SF-36)

[9] Blauwet C. Promoting the Health and Human Rights of Individuals with a Disability through the Paralympic Movement. 2005;

[10] Yazicioglu K, Yavuz F, Goktepe AS, Tan AK. Influence of adapted sports on quality of life and life satisfaction in sport participants and non-sport participants with physical disabilities. Disability and Health Journal [Internet]. Elsevier Inc; 2012;5:249-53. Available from: http://dx.doi.org/10.1016/j.dhjo.2012. 05.003

[11] Kennedy P, Rogers BA. Anxiety and depression after spinal cord injury: A longitudinal analysis. Archives of Physical Medicine and Rehabilitation. 2000; 81:932-7.

[12] Mcveigh SA, Ma SLH, Craven BC, Sj M. Influence of Sport Participation on Community Integration and Quality of Life : A Comparison between Sport Participants and Non-Sport Participants With Spinal Cord Injury Influence of Sport Participation on Community Integration. 2016;0268.

[13] O’Brien BC, Harris IB, Beckman TJ, Reed DA, Cook DA. Standards for reporting qualitative research: A synthesis of recommendations. Academic Medicine. 2014; 89:1245-51.

[14] CICONELLI RM. Tradução para o português e validação do questionário genérico de avaliação de qualidade de vida "Medical Outcomes Study 36-Item Short-Form Health Survey (SF-36)." Tese (doutorado) Universidade Federal de São Paulo [Internet]. 1997;01-120. Available from: http://www .repositorio.unifesp.br/bitstream/handle/11600/15360/Tese-3099.pdf?sequence=1\&isAllowed=y

[15] Zhang Y, Bo Q, Lun S si, Guo Y, Liu J. The 36-item short form health survey: Reliability and validity in Chinese medical students. International Journal of Medical Sciences. 2012; 9:521-6.

[16] Fawkes C. SF-36v2 is currently available in more than 170 translations. National Council For Osteopathic Research. 2013;

[17] Zhu YX, Li T, Fan SR, Liu XP, Liang YH, Liu P. Health-related quality of life as measured with the Short-Form 36 (SF-36) questionnaire in patients with recurrent vulvovaginal candidiasis. Health and Quality of Life Outcomes [Internet]. Health and Quality of Life Outcomes; 2016;14:1-6. Available from: http://dx.doi.org/10.1186/s12955-016-0470-2

[18] Tweedy SM, Beckman EM, Geraghty TJ, Theisen D, Perret C, Harvey LA, et al. Exercise and sports science Australia (ESSA) position statement on exercise and spinal cord injury. Journal of Science and Medicine in Sport [Internet]. Sports Medicine Australia; 2017; 20:108-15. Available from: http://dx.doi.org/10.1016/j.jsams.2016.02.001

[19] Nas K. Rehabilitation of spinal cord injuries. World Journal of Orthopedics. 2015; 6:8.

[20] Arango-Lasprilla JC, Nicholls E, Olivera SL, Perdomo JL, Arango JA. Health-related quality of life in individuals with spinal cord injury in Colombia, South America. NeuroRehabilitation. 2010;27:313-9.

[21] Kljajic D, Eminovic F, Dopsaj M, Pavlovic D, Arsic S, Otasevic J. The Impact Of Sports Activities On Quality Of Life Of Persons With A Spinal Cord Injury: VPLIV SPORTNIH AKTIVNOSTI NA KAKOVOST ZIVLJENJA OSEB S POSKODBO HRBTENJACE. Zdr Varst [Internet]. 2016; 55:94-101. Available from: http://www.ncbi.nlm.nih.gov/pubmed/27284378

[22] Moreira NB, Mazzardo O, Vagetti GC, Oliveira V de, Campos W de. Associação entre dependência do exercício físico e percepção da qualidade de vida no basquetebol master brasileiro. Revista Brasileira de Ciencias do Esporte. 2017; 39:433-41.

[23] Hetz SP, Latimer AE, Martin Ginis KA. Activities of daily living performed by individuals with SCI: Relationships with physical fitness and leisure time physical activity. Spinal Cord [Internet]. Nature Publishing Group; 2009; 47:550-4. Available from: http://dx.doi.org/10.1038/sc.2008.160

[24] Blauwet C, Willick SE. The paralympic movement: Using sports to promote health, disability rights, and social integration for athletes with disabilities. PM and R. 2012; 4:851-6.

[25] CAMPBELL E. Psychological Well-Being of Participants in Wheelchair Sports: Comparison of Individuals With Congenital and Acquired Disabilities. Perceptual and Motor Skills. 2011; 81:563-8.

[26] Silveira SL, Ledoux T, Cottingham M, Hernandez DC. Association among practice frequency on depression and stress among competitive US male wheelchair rugby athletes with tetraplegia. Spinal Cord [Internet]. Nature Publishing Group; 2017; 55:957-62. Available from: http://dx.doi.org/10.1038/sc. 2017.40

[27] McVeigh SA, Hitzig SL, Craven BC. Influence of sport participation on community integration and quality of life: A comparison between sport participants and non-sport participants with spinal cord injury. Journal of Spinal Cord Medicine. 2009; 32:115-24.

[28] Fiorilli G, Iuliano E, Aquino G, Battaglia C, Giombini A, Calcagno G, et al. Mental health and social participation skills of wheelchair basketball players: A controlled study. Research in Developmental 
Comparative Study of the Psychological Well-Being of Healthy Volunteers, Paralympic Athletes and NonAthletes with Spinal Cord Injury, Assessed by Short Form Survey (SF-36)

Disabilities [Internet]. Elsevier Ltd; 2013; 34:3679-85. Available from: http://dx.doi.org/10.1016/j.ridd .2013. 08.023

[29] Khazaeipour Z, Taheri-Otaghsara S-M, Naghdi M. Depression Following Spinal Cord Injury: Its Relationship to Demographic and Socioeconomic Indicators. Topics in Spinal Cord Injury Rehabilitation. 2015; 21:149-55.

Citation: Mirna Clemente, et.al. "Comparative Study of the Psychological Well-Being of Healthy Volunteers, Paralympic Athletes and Non-Athletes with Spinal Cord Injury, Assessed by Short Form Survey (SF-36)" International Journal of Sports and Physical Education (IJSPE), vol 5, no. 2, 2019, pp. 23-29. doi:http://dx.doi.org/10.20431/2454-6380.0502003.

Copyright: (c) 2019 Authors. This is an open-access article distributed under the terms of the Creative Commons Attribution License, which permits unrestricted use, distribution, and reproduction in any medium, provided the original author and source are credited. 